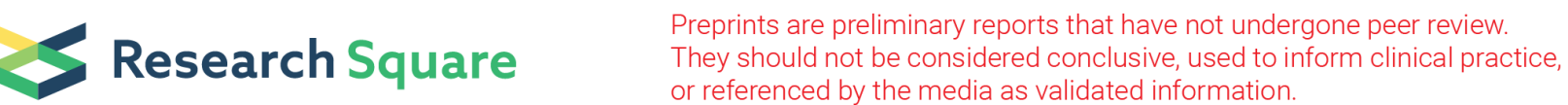

\section{Progression in time of dentate gyrus granule cell layer widening due to excitotoxicity occurs along in vivo LTP reinstatement and contextual fear memory recovery}

\section{Karina Hernández Mercado}

Universidad Nacional Autonoma de Mexico Instituto de Investigaciones Biomedicas

\section{Araceli Martinez-Moreno}

Universidad Nacional Autonoma de Mexico

\section{Luis F Rodriguez-Duran}

Universidad Nacional Autonoma de Mexico Instituto de Fisiologia Celular

Martha L. Escobar

Universidad Nacional Autonoma de Mexico

Angelica Zepeda ( $\square$ azepeda@biomedicas.unam.mx )

Universidad Nacional Autonoma de Mexico https://orcid.org/0000-0003-0857-1652

\section{Research Article}

Keywords: Brain damage, granule cell dispersion, brain plasticity, functional recovery, anxiety.

Posted Date: February 22nd, 2022

DOI: https://doi.org/10.21203/rs.3.rs-1344273/v1

License: (c) (i) This work is licensed under a Creative Commons Attribution 4.0 International License.

Read Full License 


\section{Abstract}

The dentate gyrus (DG) is the gateway of sensory information arriving from the perforant pathway (PP) to the hippocampus. The adequate integration of incoming information into the $D G$ is paramount in the execution of hippocampal-dependent cognitive functions. An abnormal DG granule cell layer (GCL) widening due to granule cell dispersion has been reported under hyperexcitation conditions in animal models as well as in patients with mesial temporal lobe epilepsy, but also in patients with no apparent relation to epilepsy. Strikingly, it is unclear whether the presence and severity of GCL widening along time affect synaptic processing arising from the PP and alter the performance in hippocampal-mediated behaviors. To evaluate the above, we injected excitotoxic kainic acid (KA) unilaterally into the DG of mice and analyzed the evolution of GCL widening at 10 and 30 days post-injection (dpi), while analyzing if KAinduced GCL widening affected in vivo long-term potentiation (LTP) in the PP-DG pathway, as well as the performance in learning and memory through contextual fear conditioning. Our results show that at 10 dpi, when a subtle GCL widening was observed, LTP induction, as well as contextual fear memory, were impaired. However, at $30 \mathrm{dpi}$ when a pronounced increase in GCL widening was found, LTP induction and contextual fear memory were already reestablished. These results highlight the plastic potential of the DG to recover some of its functions despite a major structural alteration such as abnormal GCL widening.

\section{Introduction}

The dentate gyrus (DG) is the hippocampal subfield through which sensory information coming from the entorhinal cortex (EC) enters the hippocampus (Bird and Burgess, 2008). Granule cells from the DG are activated by excitatory synaptic inputs from the entorhinal cortex via the perforant pathway (PP) (Witter, 2007; Knierim et al., 2014). The adequate DG function is critical for information processing coming from the PP and for the execution of hippocampal-dependent cognitive behaviors (Leutgeb et al., 2007). Importantly, the DG is particularly susceptible to suffer structural alterations under pathological hyperexcitation conditions (Bouilleret et al., 1999). An abnormal granule cell layer (GCL) widening due to granule cell dispersion (GCD) has been reported to occur within the normal spectrum of anatomical variation (Roy et al. 2020) but also in patients with mesial temporal lobe epilepsy and in sudden unexplained deaths in infants (Houser, 1990; Kinney et al., 2015). In this condition, granule cell bodies are abnormally separated and ectopically located within the molecular layer provoking the GCL widening (Houser, 1990).

The intrahippocampal injection of kainic acid (KA), a glutamate agonist, reproduces most of the alterations in the DG observed under pathological hyperexcitation conditions (Bouilleret et al., 1999). It has been reported that CGL widening due to GCD gradually develops after seizure activity of long duration early after intrahippocampal KA injection in mice (Tulke et al., 2019). Some studies suggest that GCD is involved in seizure generation and cognitive alterations given that the ectopic location of granule cells may represent a substrate of recurrent excitation (Cavazos et al., 2003; Pallud et al., 2011). However, findings from other studies show that granule cells surviving in zones of GCD, downscale their intrinsic excitability, mainly due to an increase in $\mathrm{K}^{+}$conductance (Stegen et al., 2009; Young et al., 2009). This 
intrinsic rescaling of granule cells has been suggested to represent an adaptive mechanism in GCD zones that likely allows to counterbalance hyperexcitability thus maintaining some DG functions under pathological hyperexcitation conditions (Yim et al., 2015).

We have previously reported that a subepileptogenic injection of KA directly into the upper DG blade of adult rats leads to a focal lesion, which shrinks in time. The decrease in the volume of the focal lesion occurs along the recovery of DG behavioral and synaptic functions. However, in our rat model, GCL widening due to GCD was not a salient feature (Zepeda et al., 2013; Aguilar-Arredondo and Zepeda, 2018). Therefore, whether DG function and hippocampal-mediated cognition are affected by the presence and severity of GCL widening along time is yet to be clarified. In this study we aimed at evaluating to what extent does the presence and severity of KA-induced GCL widening impact on DG-information processing arising from the PP and on hippocampal-mediated cognitive behaviors. To this end, we infused KA unilaterally into the upper blade of the mouse DG, where GCL widening has been shown to be consistently induced (Bouilleret et al., 1999) and analyzed the time course of GCL widening development and its association with long-term potentiation induction and contextual fear memory performance. We hereby show that GCL widening increases in time while DG functions become reinstated. Our results highlight the plastic potential of the DG as some initially lost functions due to excitotoxicty are recovered despite structural alterations such as GCL widening.

\section{Materials And Methods}

\section{Animals}

Adult C57BL/ 6 male mice 8 week-old were used throughout the experiment $(n=68)$. Mice were housed four to five per cage with free access to water and food and were kept in an inverted $12 \mathrm{~h}$ artificial light/dark cycle under standard conditions. All animal procedures were performed in agreement with local government rules (Official Mexican Standard NOM-062-ZOO-1999). Experimental protocols were approved by the animal care committee at the Instituto de Investigaciones Biomédicas-Universidad Nacional Autónoma de México (UNAM). Efforts were made to minimize animal suffering and to reduce the number of subjects used.

\section{Experimental Groups}

For behavioral and histological analysis, mice were randomly assigned to the following groups: intact ( $\mathrm{n}$ $=8), 10$ days post-sham surgery ( $10 \mathrm{dsh} ; n=11), 30$ days post-sham surgery ( $30 \mathrm{dsh} ; n=9), 10$ days postKA injection surgery ( $10 \mathrm{dpi} ; \mathrm{n}=9)$ and 30 post-KA injection surgery ( $30 \mathrm{dpi} ; \mathrm{n}=10)$. For in vivo electrophysiological recordings in the perforant pathway, independent mice not exposed to behavioral testing were used and assigned to similar groups: intact $(n=4), 10$ dsh $(n=4), 30$ dsh $(n=4), 10$ dpi $(n=$ 4) and $30 \mathrm{dpi}(\mathrm{n}=4)$.

Experimental design. Kainic acid injections or sham surgeries were performed on day 0 . Mice were handled and habituated to the experimenter for three consecutive days before the onset of behavioral 
procedures. On days 8 or 28 after surgery, mice performed the open field task. The next day (day 9 or 29) mice underwent contextual fear conditioning (CFC), and $24 \mathrm{~h}$ later, contextual fear memory (CFM) was evaluated. Immediately after memory retrieval, subjects were sacrificed and brains were extracted for histological analysis. A different group of animals, not exposed to behavioral evaluation, were used for in vivo LTP recordings (Fig. 1).

\section{Stereotaxic injection of KA and induction of DG excitotoxic damage}

We induced a focal excitotoxic lesion in the DG through the unilateral administration of a kainic acid (KA) solution. Each mouse was anaesthetized with $2-3 \%$ isoflurane in a mixture of $95 \% \mathrm{O}_{2} / 5 \% \mathrm{CO}_{2}$, and placed in a stereotaxic system (Stoelting, Wood Dale, IL). The scalp was disinfected, incised, and retracted to expose the skull. The right dorsal DG of the hippocampus was located relative to the Bregma: anteroposterior (AP), -2 mm; mediolateral (ML), $1.3 \mathrm{~mm}$; dorsoventral (DV), - $2 \mathrm{~mm}$. A circular craniotomy was performed in the mentioned coordinates, and the dura was carefully sliced with the tip of a syringe needle to allow the cannula insertion. We injected $0.15 \mu \mathrm{l}$ of a $0.75 \mathrm{mM} \mathrm{KA}$ solution (see, kainic acid solution; Sigma-Aldrich, Chemie, St. Louis, MO) at a rate of $0.15 \mu \mathrm{l} / \mathrm{min}$ through a $10 \mu \mathrm{l}$ Hamilton micro syringe mounted on a microinjection pump (Stoelting, Co., Wood Dale, IL, USA). For the sham group, the cannula was placed in the same coordinates under identical surgical conditions, but no solution was delivered. In both cases the cannula was left in the place for two min and was then slowly withdrawn; this prevented the reflux of the KA solution. The skin was sutured, and anesthesia was discontinued. Following recovery from anesthesia, mice were kept under observation for 3-4 hours. During this period, mice which received the KA injection experienced behavioral status epilepticus characterized by convulsive movements, rotations, and immobility as previously described (Riban et al., 2002). Only mice that showed behavioral status epilepticus after KA administration were kept for further experiments. The animals were left in their respective acrylic cages until the behavioral testing or electrophysiological recordings were performed.

\section{Kainic acid solution}

Kainic acid (KA, K0250; Sigma-Aldrich, Chemie, St. Louis, MO Sigma-Aldrich, MO) was dissolved in $1 \mathrm{~N}$ $\mathrm{NaOH}$, and the solution was brought to the desired volume with $10 \mathrm{mM}$ phosphate buffer $(\mathrm{pH} 7.2)$ for a final $0.75 \mathrm{mM}$ solution. The $\mathrm{pH}$ was adjusted to 7.0 with $2 \mathrm{~N} \mathrm{HCl}$. A fluorescence tracer (red Retrobeads, LumaFluor, Naples, FL) was added to the stock KA solution $((49 \mu \mathrm{l}$ of KA $(0.75 \mathrm{mM})+1 \mu \mathrm{l}$ of fluorescence tracer) for proper visualization of the injection site and KA spreading within the GCL.

\section{Histological procedures}

Mice were deeply anesthetized with an overdose of sodium pentobarbital $(210 \mathrm{mg} / \mathrm{kg})$ and transcardially perfused with ice-cold $0.9 \%$ saline solution followed by ice-cold $4 \%$ paraformaldehyde in $0.1 \mathrm{M}$ phosphate buffer ( $\mathrm{pH}$ 7.4). Next, brains were removed, post-fixed in paraformaldehyde for $24 \mathrm{~h}$, and were then successively transferred to $15 \%$ and $30 \%$ sucrose in $0.1 \mathrm{M}$ phosphate buffer. We obtained $40-\mu \mathrm{m}$ thick 
coronal brain sections using a cryostat (Microm HM550, Thermo Fisher Scientific, Waltham, MA, USA). One in every three sections was mounted on gelatin-covered slides for Nissl staining.

\section{Nissl staining}

Nissl staining was performed to analyze the gross morphology of the DG in all groups. The DG volume, as well as the volume of the focal lesion and the GCL width from the injected DG was measured. Sections were mounted on gelatine-covered slides and rehydrated with distilled water. Then sections were dehydrated in a series of alcohol solutions (70, 95 and 100\%, 2 min in each) and immersed in xylol. Sections were then rehydrated, immersed in $0.1 \%$ cresyl violet (Sigma-Aldrich, St. Louis, MO, USA) and dehydrated before being covered with Permount (Fischer Scientific, NJ, USA) and a coverslip. Sections were captured with a 20X objective using a disk scanning unit microscope (Olympus BX51WI).

\section{Measurement of the GCL width}

To evaluate the impact of the KA injection on GCL widening, the average width of the GCL was determined from Nissl-stained sections in all experimental groups following the procedures described in Müller et al., 2009 using the Stereolnvestigator software (MBF Bioscience, Inc., Williston, VT).

Briefly, the brain section showing the maximal GCL widening from the dorsal DG ipsilateral to the injection, was chosen as a start point for the measurements. We analysed 4-5 consecutive sections from the "start section" (each section separated by $80 \mu \mathrm{m}$ from the next) and measured the GCL width (in the dorso-ventral plane) at regular $100 \mu \mathrm{m}$ intervals in the mediolateral (ML) axis along the upper and lower blades from each section. In all cases, a total length of $520 \mu \mathrm{m}$ in the anteroposterior axis were analyzed. For calculating the dimension of the focal lesion zone produced in the upper blade, measurements were performed at shorter intervals of $50 \mu \mathrm{m}$ (in the mediolateral axis) due to the reduced blade area. For statistical analysis, the mean width from each DG analyzed section and region (i.e. upper blade, lower blade and lesion area) was calculated.

\section{Volumetric analysis of the DG and the focal lesion zone}

The volume of the dentate granule cell layer from Nissl-stained sections was calculated using the Cavalieri estimator stereological method (Stereolnvestigator software; MBF Bioscience, Inc., Williston, VT) as previously described (Aguilar-Arredondo and Zepeda, 2018). Six sections per brain were used, each one was separated from the next by $80 \mu \mathrm{m}$ within AP $-1.94 \mathrm{~mm}$ and $-2.46 \mathrm{~mm}$. The contour of the DG from all sections was delineated, the volume of the granule layer from each subject was automatically calculated, and the average per group was obtained and reported in $\mathrm{mm}^{3}$. The volume of the focal lesion zone produced in the upper blade was obtained using the same method by delineating the thinned area in five sections per brain.

\section{In vivo electrophysiological recordings \\ Long-term potentiation (LTP) in the PP-DG}


Mice were anesthetized with pentobarbital $(50 \mathrm{mg} / \mathrm{kg})$, and their temperature was maintained with gel thermal pads. Mice were fixed on a stereotaxic frame, and the skull was exposed. Unilateral responses were registered with a stainless-steel monopolar electrode located in the dentate gyrus (DG) in the coordinates: AP - 2.0 from Bregma, ML - 1.2 from the midline and DV - 1.8 from the dura (Huang et al. 2012; Nelson-Mora et al., 2018). Responses were unilaterally evoked by a bipolar concentric stainlesssteel stimulation electrode (100- $\mu \mathrm{m}$ diameter) located in the perforant pathway (PP) at the following coordinates: AP - 0.2 from Lambda, ML - 2.8 from the midline and DV - 1.5 from dura. Constant (400$800 \mu \mathrm{A}$ monophasic pulse, $0.25 \mathrm{~ms}$ ) duration current stimulation was provided by a Grass S48 stimulator and delivered to the stimulating electrode through a Grass Stimulus Isolation Unit (PSUI6). Evoked responses were sent to an amplifier Grass P5. The electric signal was digitalized, stored, and analyzed using the software Datawave SciWorks (Broomfield, CO, USA). Baseline was established after 20 min of evoked responses. The applied stimulus intensity corresponded to $50 \%$ of the excitatory post-synaptic potential (EPSP) maximum amplitude. LTP was induced by delivering 5 high-frequency conditioning stimulus trains, each consisting of 10 bursts of 20 pulses each $(400 \mathrm{~Hz}$ with $15 \mathrm{~s}$ of inter-train time (Nelson-Mora et al., 2018).

\section{Behavioral testing}

The open field task was conducted on days 8 or 28 post-KA injection or sham procedures to analyze anxiety and general motor performance. This test was followed by contextual fear conditioning (CFC) on days 9 or 29, and contextual fear memory (CFM) on days 10 or 30 after surgical procedures (see, Fig. 1). Mice were handled and habituated to the experimenter for three consecutive days before behavioral procedures were performed. Thirty minutes previous to behavioral evaluation, mice were habituated to the testing room.

\section{Open field test}

Mice were evaluated in the open field arena to analyze anxiety and general motor performance one day before CFC. The open field consisted on a white acrylic arena $40 \mathrm{~cm}$ wide $X 40 \mathrm{~cm}$ length $X 25 \mathrm{~cm}$ height. To facilitate the analysis of locomotion and the time spent in the center and periphery zones, the floor was divided into 16 drawn squares $(10 \mathrm{~cm} \times 10 \mathrm{~cm}$ each square) where 4 were central and 12 were peripheral. Each mouse was individually placed in a corner of the arena and was allowed to explore freely. Behavior was videotaped for 5 minutes. The total number of line crossings (from one square to another) and the time spent in the center or periphery zones during 5 min were recorded. Caution was taken to counterbalance the introduction of the mice to each of the four corners of the arena. Before every trial, the arena was wiped with a cleaning solution consisting of $10 \%$ EtOH and $10 \%$ Extran (Merck, Darmstadt, Germany) diluted in distilled water.

\section{Contextual fear conditioning and memory}

The task was performed in a mouse conditioning chamber measuring $25 \mathrm{~cm} \times 25 \mathrm{~cm}$ length and $20 \mathrm{~cm}$ height (San Diego Instruments, San Diego, CA, USA). The chamber consisted of transparent acrylic walls 
and 23 stainless steel floor rods. The system was equipped with a matrix of $16 \times 16$ infrared beams at the floor level. Movements inside the chamber were registered by the interruption of a beam and were recorded with the aid of the freeze monitor software (Freeze Monitor, SD Instruments). Movements were also recorded by an observer and were compared to those recorded by the computer. The contextual fear conditioning (CFC) phase was performed on day 9 or 29 after KA injection or after sham surgery. Mice were placed individually in the conditioning chamber and were allowed to explore it for $2 \mathrm{~min}$ before the onset of the first foot shock (pre-shock time). After this lapse, five foot shocks ( $2 \mathrm{~s}, 0.5 \mathrm{~mA}$ ) were delivered at variable intervals ranging from 30 to 180 seconds. The entire training session lasted $12 \mathrm{~min}$, and the movement of each mouse was evaluated along the whole session. Twenty-four hours later, subjects were evaluated for contextual fear memory by placing each mouse in the conditioning chamber in the absence of foot shock, and their freezing time was recorded for $5 \mathrm{~min}$. Subjects were then returned to their home cages. Before each session, the chamber was wiped with a cleaning solution consisting of $10 \% \mathrm{EtOH}$ and 10\% Extran (Merck, Darmstadt, Germany) diluted in distilled water.

\section{Statistical analysis}

Data were analyzed using GraphPad Prism 7 software. One-way ANOVA followed by a Bonferroni's post hoc test was used to analyze data from: the Cavalieri estimation of DG volume, the number of line crossings in the open field, and immobility time for the contextual fear memory task. An unpaired t-test with Welch's correction was used to analyze the Cavalieri estimation of the lesion volume in the DG upper blade and to analyze the obtained measurements of DG width in the upper and lower blades. Two-way ANOVA followed by a Bonferroni's post hoc test was performed to analyze the time spent in the periphery and center zones in open field, and time of immobility during contextual fear conditioning. A repeatedmeasures ANOVA followed post hoc Fisher's test was used to analyze LTP results. Data evaluated through ANOVA passed the normality and equality variance tests. $p<0.05$ was considered statistically significant in all cases.

\section{Results}

\section{Morphological changes over time in the DG after a focal injection of KA}

We injected KA unilaterally in the upper blade of the dorsal DG and induced a focal excitotoxic lesion in DG along with GCL widening. In order to analyze the evolution of KA injection upon the DG structure, we evaluated the gross morphological changes of the DG blades as well as the evolution of the focal lesion at 10 and 30 days post-KA injection (dpi; Fig. 1). The DG of sham mice at 10 days post sham surgery (10 dsh) showed mechanical damage provoked by the cannula insertion in the upper blade (Fig. 2A, arrowhead), which was not evident in the 30 dsh group (Fig. 2E, head arrow). None of the sham groups showed GCL widening. However, a progressive increase in GCL widening was observed from $10 \mathrm{dpi}$ to 30 dpi in the KA-injection groups (Fig. 2B and F). Mice developed GCL widening in the injected DG but not in the contralateral side (data not shown). GCL widening consisted on the increase in the volume and width 
of both DG blades due to granule cell distant apposition (Fig. 2 l and K, $10 \mathrm{dpi}<30 \mathrm{dpi}, \mathrm{p}<0.0001$ ). Still, in the lower blade, this effect was consistently stronger than in the upper blade where the KA-induced focal lesion was located (Fig. 2, volume in 2l, 30 dpi: upper (mean \pm SD) $0.2536 \mathrm{~mm}^{3} \pm 0.03984$ vs lower 0.3352 $\mathrm{mm}^{3} \pm 0.04337, \mathrm{p}=0004$, and width in $\mathrm{K}, 10 \mathrm{dpi}$ : upper $60.64 \mu \mathrm{m} \pm 14.63$ vs lower $107.3 \mu \mathrm{m} \pm 14.58,30$ dpi: upper $80.03 \mu \mathrm{m} \pm 27.85$ vs lower $188.8 \mu \mathrm{m} \pm 37.16, \mathrm{p}<0.0001)$. At $10 \mathrm{dpi}$, the KA-induced focal lesion was characterized by a thinning of the granule cell layer due to the cell loss (decreased layer volume and width), but at $30 \mathrm{dpi}$, the lesion site had widened probably as a result of the dispersion of the surviving granule cells (Fig. 2, white arrow in B and F; blue colored zone in $\mathrm{D}$ and $\mathrm{H}$; volume in $\mathrm{J}, 10$ dpi: 0.01083 $\mathrm{mm}^{3} \pm 0.00162$ vs $30 \mathrm{dpi}: 0.03024 \mathrm{~mm}^{3} \pm 0.00695, \mathrm{p}<0.0027$; width of focal lesion in $\mathrm{K}, 10 \mathrm{dpi}: 22.37$ $\mu \mathrm{m} \pm 5.97$ vs $30 \mathrm{dpi}: 43.84 \mu \mathrm{m} \pm 11.88, \mathrm{p}<0.0001$ ). This KA-induced focal lesion co-localized with the area where the KA spread, as revealed by the fluorescent tracer (Fig. $2 \mathrm{C}$ and $\mathrm{G}$ ). The volume of the DG blades were similar across time between sham and intact groups (Fig. 2l, total GCL in intact: $0.2156 \mathrm{~mm}^{3}$ $\pm 0.0295,10$ dsh: $0.2209 \mathrm{~mm}^{3} \pm 0.0199,30$ dsh: $0.2411 \mathrm{~mm}^{3} \pm 0.0137$, no significant (ns) $p>0.9999$ ). Figure $2 \mathrm{~L}$ shows the average volume and Gundersen's coefficient of the DG blades and the KA-induced focal lesion obtained after a stereological Cavalieri analysis.

In addition to the morphological alterations described above, KA-injected mice also showed cell loss in the hilus, as well as in the CA3 and CA1 hippocampal sub-regions. The cell loss observed in CA1 was consistent, but of variable severity among mice. Summarizing, in KA-injected mice, we observed three main features: 1) a progressive increase of GCL widening over time, being more pronounced at $30 \mathrm{dpi}$ than at $10 \mathrm{dpi}$; 2) a focal lesion in the upper blade where KA diffused; and 3) cell loss in the hilus, as well as in the CA3 and CA1 hippocampal sub-regions.

\section{Perforant pathway- dentate gyrus long-term potentiation in vivo}

In order to evaluate the functional impact of KA-induced alteration on DG-information processing arising from the PP, we analyzed the establishment of LTP in the perforant pathway-dentate gyrus (PP-DG) using the protocol depicted in Fig. 3A. Figure 3B shows representative traces of the excitatory post-synaptic potential (EPSP) obtained during baseline (dotted line) and 140 min after stimulation (full line). After high-frequency stimulation, all groups displayed LTP, except for the 10 dpi group, in which LTP was impaired (Fig. 3C, D). LTP was similar in intact and 30 dsh mice showing a 71 and $72 \%$ EPSP slope increase, respectively. Still, mice from the 10 dsh group showed a more discrete EPSP slope increase of only $40 \%$ without a progressive rise in magnitude along the recording (Fig. $3 \mathrm{C}$ ). At $30 \mathrm{dpi}$, the ability to produce LTP was restored, though with a discrete EPSP slope increase of $51 \%$, which was lower than that observed in intact and 30 dsh groups (Fig. 3C). Notably, LTP from 30 dpi subjects took longer to reach its maximal response compared to all other groups. The difference in the slope between the $30 \mathrm{dpi}$ vs control groups (intact and $30 \mathrm{dsh}$ ) and $10 \mathrm{dsh}$ vs control groups was statistically significant (repeated-measures ANOVA differences $F_{(4,15)}=133.64, p<0.0001$, and post hoc Fisher's test $\left.p<0.0001\right)$. We also analyzed the population spike slope change and similar results were found as for the EPSP slope. Indeed, the trend 
was the same, although differences were more pronounced in the population spike slope as expected (Fig. 3D, repeated-measures ANOVA differences $F_{(4,15)}=549.84, p<0.0001$ and post hoc Fisher's $p<$ 0.0001). Therefore, in KA-injected mice, LTP induction was impaired at $10 \mathrm{dpi}$, but at $30 \mathrm{dpi}$ the ability to elicit LTP was restored, though with a lower increase percentage than in control mice.

\section{Mice at $10 \mathrm{dpi}$ and $\mathbf{3 0} \mathrm{dpi}$ show increased locomotor activity and anxiety like behavior}

Mice were evaluated in an open field task to estimate their locomotor performance and anxiety behavior. During the behavioral assessment none of the mice displayed behavioral seizures. We observed that KAinjected mice at $10 \mathrm{dpi}$ had an increased motility and anxiety-like behavior, which persisted at $30 \mathrm{dpi}$. Motility was assessed through the number of line crossings along the open field test. KA-injected groups displayed similar number of line crossings, which were higher than in the control group (intact and sham) (Fig. 4A; 10 dpi: $195.5 \pm 19.87$ and 30 dpi: $191.5 \pm 23.67$ > intact: $122.4 \pm 25.46,10$ dsh; $139.1 \pm 21.14$, and 30 dsh: $137.3 \pm 19.9, p<0.0001)$. Anxiety behavior was evaluated through the time spent in the periphery

and center zone of the arena. KA-injected mice at 10 and $30 \mathrm{dpi}$ spent more time in the periphery and less time in center zone than control mice, which reflected an anxious behavior (Fig. 4B-C; two-way ANOVA followed by a Bonferroni's post hoc test, interaction $\left.F_{(4,82)}=16.18, p<0.0001\right)$. Therefore, KA-injected mice showed augmented motility and anxiety-like behavior, which lasted from10 to $30 \mathrm{dpi}$.

\section{Contextual fear conditioning is initially impaired and restored along time}

We next asked if the development over time of GCL widening had a negative impact on a hippocampaldependent behavior. To address this question, we evaluated the performance of mice in contextual fear conditioning (CFC), a hippocampal-dependent cognitive task (Lee and Kesner, 2004; Bernier et al., 2017). All mice, except the $10 \mathrm{dpi}$ group, exhibited progressive learning during conditioning, which was reflected by the continuous increase in freezing behavior across trials (Fig. 5A; two-way ANOVA followed by a Bonferroni's post hoc test, $\left.F_{(4,192)}=43.07, p<0.0001\right)$. This indicates that KA-injected mice at $10 \mathrm{dpi}$, but not at $30 \mathrm{dpi}$ were impaired in this learning paradigm. Contextual fear memory was evaluated $24 \mathrm{~h}$ after conditioning, and the $10 \mathrm{dpi}$ group displayed less freezing time than mice from all other groups, denoting impairment in contextual fear learning and memory (Fig. 5B; 10 dpi: $11.54 \pm 4.623<$ intact: $67.43 \pm 9.88$, 10 dsh: $33.88 \pm 9.151,30$ dsh: $59.64 \pm 14.59$, and 30 dpi: $57.07 \pm 19.78$ ). However, at 30 dpi KA-injected mice had increased their freezing response exhibiting values similar to intact and $30 \mathrm{dsh}$ mice, which evidences a contextual fear memory recovery (Fig. 5B; 30 dpi $57.07 \pm 19.78$ vs intact $67.43 \pm 9.88$ and 30 dsh $59.64 \pm 14.59$, ns $p>0.9999$ ). These results are in line with our electrophysiological observations where LTP was impaired at $10 \mathrm{dpi}$ and restored and $30 \mathrm{dpi}$. Concerning contextual fear memory retrieval in mice from the $10 \mathrm{dsh}$ group, they displayed a diminished percentage in freezing time than mice from intact and 30 dsh (Fig. 5B; 10 dsh $33.38 \pm 9.15<$ intact $67.43 \pm 9.88$, and 30 dsh $59.64 \pm 14.59$ ). The lower freezing observed in the 10 dsh group correlated with the lower LTP response and the mechanical lesion observed in Nissl stained tissue due to the cannula insertion. The mild impairment in LTP as well as the 
cannula-induced damage were completely recovered in the 30 dsh group, and the freezing percentage was similar to that observed in intact animals (Fig. 5B; 30 dsh: $59.64 \pm 14.59$ vs intact: $67.43 \pm 9.88$, ns p $>0.9999)$.

Given that GCL widening increased along time, as did the freezing percentage in CFM, we evaluated if the development of GCL widening at 10 and 30 dpi correlated with the percentage in freezing time observed during memory recall. We found a positive correlation between the increase in the volume of the lower blade (reflecting GCL widening) as well as in the upper blade and the increase in freezing percentage across time (Fig. 5C and D; Spearman correlation coefficient).

Overall these results show that the progressive GCL widening caused by exitotoxicity does not hinder the recovery of a hippocampal-dependent cognitive behavior and of LTP in the PP-DG pathway.

\section{Discussion}

We evaluated how hippocampal cognitive behavior and synaptic plasticity in the PP-DG pathway adapt along the gradual increase of GCL widening after focal unilateral KA-injection in the DG upper blade. We found that the gradual increase in GCL widening did not associate with a decrease in cognitive behavior and synaptic plasticity along time. Impaired cognitive behavior and LTP in the PP-DG pathway were observed to occur along a mild increase in GCL widening at 10 days post-KA injection (dpi). However, the recovery of cognitive behavior and improved LTP occurred concomitant with a pronounced increase in GCL widening at $30 \mathrm{dpi}$.

We found that after inducing a KA focal lesion in the DG, a similar progressive development of GCL widening occurred as previously reported in other studies using a mouse epilepsy model where widening began to be visible after 1 week of KA-injection and reached its peak after 3 weeks (Chai et al., 2014; Janz et al., 2017). This time window allowed us to study the impact of GCL widening development in the DG function: at $10 \mathrm{dpi}$ when the widening was readily visible and at $30 \mathrm{dpi}$ when it was fully developed and our results provide evidence that the DG reinstates some of its intrinsic functions despite GCL widening. The present work differs from studies using the KA-mouse epilepsy model, where KA is injected close to the hippocampal fissure to induce complete GCD on both DG blades (Heinrich, 2006). In our study, the lower blade disperses completely while the upper blade shows a slight dispersion mainly in the surroundings of the focal lesion where abundant cell loss was found. We reproduced the rat focal lesion in the upper blade of DG, where LTP (mossy fiber pathway) and cognitive behavior were impaired at 10 dpi, but recovered at $30 \mathrm{dpi}$, although, in rat the GCD was not a salient feature (Zepeda et al., 2013; Aguilar-Arredondo and Zepeda, 2018). Thus in the present study we induced the focal lesion in mouse where GCD is easily and gradually induced along time. An important finding in our study was that GCL widening, due to granule cell dispersion (GCD), did not impede the recovery of the hippocampaldependent cognitive behavior and LTP in the PP-DG pathway.

It has been suggested that the progressive increase in GCL widening due to GCD triggers homeostatic changes in granule cells that contribute to maintain DG-associated functions under pathological 
hyperexcitation conditions (Yim et al., 2015). However, it has also been shown that GCD is not unique to patients with epilepsy and may occur as a regular anatomical variation within humand (Roy et al., 2020). In the context of hyperexcitation, electrophysiological studies report that granule cells surviving in GCD regions, reduce their intrinsic excitability by decreasing calbindin-dependent calcium influx and increasing potassium outflux in both mesial temporal lobe epilepsy (MTLE) patients and in the KA-mouse model of MTLE (Nägerl et al., 2000; Stegen et al., 2009; Young et al., 2009). The rescaling in the excitability of granule cells points to a mechanism that could decrease hyperexcitability in GCD zones (Young et al., 2009). In line with the latter, it has been shown that GCD zones display lower intensities of seizure activity than non-GCD zones in the KA- mouse model of MTLE (Le Duigou et al., 2008; Haussler et al., 2012; Janz et al., 2018; Rougier et al., 2005). Changes occurring in GCD zones could reduce hyperexcitability in the DG and by reducing excitability under epileptic conditions, the DG may restore some of its associated functions, as previously predicted by computational models (Yim et al., 2015). Our results are in agreement with the above-described studies as the severe GCL widening found at $30 \mathrm{dpi}$ correlated in time with an improvement in LTP and cognitive behavior. This suggests that GCL widening, due to GCD, may represent a plastic adaptation that may contribute to maintain DG function in mice under pathological hyperexcitation conditions.

It should be also considered that the low activity of granule cells is important for the proper performance of DG-associated network function and hippocampal cognition (Leutgeb et al., 2007). This low activity is maintained in part through the strong inhibition exerted by GABAergic interneurons in the DG (Coulter and Carlson, 2007). During the first week after KA administration, studies report a reduction in the inhibitory efficacy in the DG. The levels of neurotransmitter GABA decrease early at day 1 after the intrahippocampal KA administration in mice and keep low within the first week (Janz et al., 2017). Moreover, the $\mathrm{E}_{\mathrm{GABA}}$ (current equilibrium potential) of granule cells shifts to less hyperpolarized levels (-55 to $-60 \mathrm{mV}$ ) during the first week after excitotoxic damage (Pathak et al., 2007). This greatly diminishes inhibitory efficacy and increases granule cells excitability. Interestingly, the levels of GABA return to normal at four weeks after the intrahippocampal KA administration in mice (Janz et al., 2017). Similarly, $E_{G A B A}$ in granule cells return to near control levels at four weeks after pilocarpine administration, which increases inhibitory efficacy in DG (Pathak et al., 2007). The restoration of inhibition in the DG is important for the establishment of proper excitation/inhibition balance. Given that restoration occurs around week four, it is possible to suggest that it contributed to the reestablishment of LTP and to the cognitive recovery observed at $30 \mathrm{dpi}$ in our study.

Our electrophysiological results show that despite the visible improvement in LTP at $30 \mathrm{dpi}$, the trace differed from that in the intact and 30 dsh groups. LTP at 30 dpi shows a lower response and a slower increase across time after high frequency stimulation (HFS). Therefore, it is likely that the mechanisms related to the early-LTP phase induction are impaired in mice at $30 \mathrm{dpi}$. These mechanisms involving the PP-DG pathway associate with the activation of AMPA and NMDA receptors (Bliss et al., 2018; Huang, 1998). In the KA-mouse model, the density of AMPA receptors is attenuated in dispersed zones, which predicts a weakening of synaptic efficacy (Janz et al., 2018). The low density of AMPA receptors may 
delay the entry of $\mathrm{Ca}^{2+}$ necessary to induce the fast increase in early-LTP response (Bliss et al., 2018). Consequently, other mechanisms like activation of metabotropic receptors and subsequent increase of cAMP may be responsible for the slow increase in LTP observed here at $30 \mathrm{dpi}$ (Nguyen and Kandel, 1996). Further studies are necessary to reveal the causes that underlie this different LTP response. However, this suggests that synaptic plasticity in PP-DG pathway is still compromised at $30 \mathrm{dpi}$, but with a visible improvement compared to $10 \mathrm{dpi}$.

Results from the 10 dsh group show a lower LTP response and freezing percentage time during memory retrieval compared to control mice (intact and $30 \mathrm{dsh}$ ). We associate these events with the interruption of the granule cell layer observed by the cannula insertion during surgery procedures. It is likely that this mechanical lesion promoted the activation of calpains, which are increased in neuronal damage resulting from traumatic injury (Saatman et al., 2010). Calpains can limit the magnitude of LTP and compromise cognition (Wang et al., 2014). Likewise, mice from the 10 dpi group may have been affected by the cannula insertion. However, this group showed a complete impairment in both evaluated DG functions reflecting the contribution of KA-induced excitotoxic damage.

Finally, one possible caveat regarding our learning and memory results is that lesioned mice show anxiety and hyperactive phenotypes in the open field test, which is in line with previous results showing that anxiety and hyperactive behaviors are still observed after one month of KA injection (Zeidler et al., 2018). It could be argued that this would complicate the interpretation on the freezing results observed in the memory task. However, while both groups, 10 and $30 \mathrm{dpi}$, exhibited similar hyperactive and anxiety behaviors, only mice from the $10 \mathrm{dpi}$ group displayed learning and memory impairments. This supports the conclusion that the hyperactive and anxiety behaviors might not account for the cognitive results observed here.

In conclusion, our results show that GCL widening does not hinder long-term DG function at a behavioral nor at a synaptic level and together with evidence provided from other groups, our results may contribute to the idea that injury-induced GCL widening following excitotoxicity is not enough to impede DG functions over time.

\section{Declarations}

\section{Competing interests}

The authors have no relevant financial or non-financial interests to disclose.

\section{Funding}

This project was supported by CONACyT 282470 (AZ) and by CONACyT 215719 (MLE). Karina Hernández Mercado is a doctoral student from Programa de Doctorado en Ciencias Biomédicas, Universidad Nacional Autónoma de México (UNAM) and was supported by CONACyT 308538. 


\section{Author contributions}

Karina Hernández: designed research, performed research, analyzed data and wrote paper; Araceli Martínez and Rodríguez Durán: performed research and analyzed data; Martha L: designed research and analytic tools; and Angélica Zepeda: designed research and wrote paper.

\section{Data availability}

Data derived from this study is available as per request to the corresponding author

\section{Ethics approval}

All animal procedures were performed in agreement with local government rules (Official Mexican Standard NOM-062-ZOO-1999). Experimental protocols were approved by the animal care committee at the Instituto de Investigaciones Biomédicas-Universidad Nacional Autónoma de México (UNAM).

\section{References}

Aguilar-Arredondo A, Zepeda A (2018) Memory retrieval-induced activation of adult-born neurons generated in response to damage to the dentate gyrus. Brain Struct Funct 223:2859-2877.

Bernier BE, Lacagnina AF, Ayoub A, Shue F, Zemelman B V., Krasne FB, Drew MR (2017) Dentate Gyrus Contributes to Retrieval as well as Encoding: Evidence from Context Fear Conditioning, Recall, and Extinction. J Neurosci 37:6359-6371.

Bird CM, Burgess N (2008) The hippocampus and memory: insights from spatial processing. Nat Rev Neurosci 9:182-194.

Bliss TVP, Collingridge GL, Morris RGM, Reymann KG (2018) Long-term potentiation in the hippocampus: discovery, mechanisms and function. Neuroforum 24:A103-A120.

Bouilleret V, Ridoux V, Depaulis A, Marescaux C, Nehlig A, Le Gal La Salle G (1999) Recurrent seizures and hippocampal sclerosis following intrahippocampal kainate injection in adult mice: electroencephalography, histopathology and synaptic reorganization similar to mesial temporal lobe epilepsy. Neuroscience 89:717-729.

Cavazos JE, Zhang P, Qazi R, Sutula TP (2003) Ultrastructural features of sprouted mossy fiber synapses in kindled and kainic acid-treated rats. J Comp Neurol 458:272-292.

Chai X, Münzner G, Zhao S, Tinnes S, Kowalski J, Häussler U, Young C, Haas CA, Frotscher M (2014) Epilepsy-Induced Motility of Differentiated Neurons. Cereb Cortex 24:2130-2140.

Coulter DA, Carlson GC (2007) Functional regulation of the dentate gyrus by GABA-mediated inhibition. In, pp 235-812. 
Haussler U, Bielefeld L, Froriep UP, Wolfart J, Haas CA (2012) Septotemporal Position in the Hippocampal Formation Determines Epileptic and Neurogenic Activity in Temporal Lobe Epilepsy. Cereb Cortex 22:2636 .

Heinrich C (2006) Reelin Deficiency and Displacement of Mature Neurons, But Not Neurogenesis, Underlie the Formation of Granule Cell Dispersion in the Epileptic Hippocampus. J Neurosci 26:4701-4713.

Hernández-Ortega K, Ferrera P, Arias C (2007) Sequential expression of cell-cycle regulators and Alzheimer's disease-related proteins in entorhinal cortex after hippocampal excitotoxic damage. $\mathrm{J}$ Neurosci Res 85:1744-1751.

Houser CR (1990) Granule cell dispersion in the dentate gyrus of humans with temporal lobe epilepsy. Brain Res 535:195-204.

Huang EP (1998) Synaptic plasticity: Going through phases with LTP. Curr Biol 8:R350-R352.

Janz P, Hauser P, Heining K, Nestel S, Kirsch M, Egert U, Haas CA (2018) Position- and Time-Dependent Arc Expression Links Neuronal Activity to Synaptic Plasticity During Epileptogenesis. Front Cell Neurosci 12. fncel.2018.00244.

Janz P, Schwaderlapp N, Heining K, Häussler U, Korvink JG, von Elverfeldt D, Hennig J, Egert U, LeVan P, Haas CA (2017) Early tissue damage and microstructural reorganization predict disease severity in experimental epilepsy. Elife 6:e25742.

Kinney HC, Cryan JB, Haynes RL, Paterson DS, Haas EA, Mena OJ, Minter M, Journey KW, Trachtenberg FL, Goldstein RD, Armstrong DD (2015) Dentate gyrus abnormalities in sudden unexplained death in infants: morphological marker of underlying brain vulnerability. Acta Neuropathol 129:65-80.

Knierim JJ, Neunuebel JP, Deshmukh SS (2014) Functional correlates of the lateral and medial entorhinal cortex: objects, path integration and local-global reference frames. Philos Trans R Soc B Biol Sci 369:20130369.

Le Duigou C, Bouilleret V, Miles R (2008) Epileptiform activities in slices of hippocampus from mice after intra-hippocampal injection of kainic acid. J Physiol 586:4891-4904.

Lee I, Kesner RP (2004) Differential contributions of dorsal hippocampal subregions to memory acquisition and retrieval in contextual fear-conditioning. Hippocampus 14:301-310.

Leutgeb JK, Leutgeb S, Moser M-B, Moser El (2007) Pattern Separation in the Dentate Gyrus and CA3 of the Hippocampus. Science (80-) 315:961-966.

Müller MC, Osswald M, Tinnes S, Häussler U, Jacobi A, Förster E, Frotscher M, Haas CA (2009) Exogenous reelin prevents granule cell dispersion in experimental epilepsy. Exp Neurol 216:390-397. 
Nägerl UV, Mody I, Jeub M, Lie AA, Elger CE, Beck H (2000) Surviving Granule Cells of the Sclerotic Human Hippocampus Have Reduced Ca 2+ Influx Because of a Loss of Calbindin-D 28k in Temporal Lobe Epilepsy. J Neurosci 20:1831-1836.

Nelson-Mora J, Escobar ML, Rodríguez-Durán L, Massieu L, Montiel T, Rodríguez VM, Hernández-Mercado K, Gonsebatt ME (2018) Gestational exposure to inorganic arsenic (iAs3+) alters glutamate disposition in the mouse hippocampus and ionotropic glutamate receptor expression leading to memory impairment. Arch Toxicol 92:1037-1048.

Nguyen P V., Kandel ER (1996) A Macromolecular Synthesis-Dependent Late Phase of Long-Term Potentiation Requiring cAMP in the Medial Perforant Pathway of Rat Hippocampal Slices. J Neurosci 16:3189-3198.

Pallud J, Häussler U, Langlois M, Hamelin S, Devaux B, Deransart C, Depaulis A (2011) Dentate gyrus and hilus transection blocks seizure propagation and granule cell dispersion in a mouse model for mesial temporal lobe epilepsy. Hippocampus 21:334-343.

Pathak HR, Weissinger F, Terunuma M, Carlson GC, Hsu F-C, Moss SJ, Coulter DA (2007) Disrupted Dentate Granule Cell Chloride Regulation Enhances Synaptic Excitability during Development of Temporal Lobe Epilepsy. J Neurosci 27:14012-14022.

Riban V, Bouilleret V, Pham-Lê BT, Fritschy J-M, Marescaux C, Depaulis A (2002) Evolution of hippocampal epileptic activity during the development of hippocampal sclerosis in a mouse model of temporal lobe epilepsy. Neuroscience 112:101-111.

Rougier A, Arthaud S, Zombre N, Salle GLG La (2005) Patterns of dentate granule cell responses to perforant path stimulation in epileptic mice with granule cell dispersion. Epilepsy Res 63:119-129.

Roy A, Millen KJ, Kapur RP (2020) Hippocampal granule cell dispersion: a non-specific finding in pediatric patients with no history of seizures. Acta Neuropathol Commun. 8(1):54.

Saatman KE, Creed J, Raghupathi R (2010) Calpain as a therapeutic target in traumatic brain injury. Neurotherapeutics 7:31-42.

Stegen M, Young CC, Haas CA, Zentner J, Wolfart J (2009) Increased leak conductance in dentate gyrus granule cells of temporal lobe epilepsy patients with Ammon's horn sclerosis. Epilepsia 50:646-653.

Tulke S, Haas CA, Häussler U (2019) Expression of brain-derived neurotrophic factor and structural plasticity in the dentate gyrus and $\langle s c p>C A</ s c p>2$ region correlate with epileptiform activity. Epilepsia:epi.15540.

Wang Y, Zhu G, Briz V, Hsu Y-T, Bi X, Baudry M (2014) A molecular brake controls the magnitude of longterm potentiation. Nat Commun 5:3051. 
Witter MP (2007) The perforant path: projections from the entorhinal cortex to the dentate gyrus. In, pp $43-61$.

Yim MY, Hanuschkin A, Wolfart J (2015) Intrinsic rescaling of granule cells restores pattern separation ability of a dentate gyrus network model during epileptic hyperexcitability. Hippocampus 25:297-308.

Young CC, Stegen M, Bernard R, Müller M, Bischofberger J, Veh RW, Haas CA, Wolfart J (2009) Upregulation of inward rectifier $\mathrm{K}+$ (Kir2) channels in dentate gyrus granule cells in temporal lobe epilepsy. J Physiol 587:4213-4233.

Zeidler Z, Brandt-Fontaine M, Leintz C, Krook-Magnuson C, Netoff T, Krook-Magnuson E (2018) Targeting the Mouse Ventral Hippocampus in the Intrahippocampal Kainic Acid Model of Temporal Lobe Epilepsy. eneuro 5:ENEURO.0158-18.2018.

Zepeda A, Aguilar-Arredondo A, Michel G, Ramos-Languren LE, Escobar ML, Arias C (2013) Functional recovery of the dentate gyrus after a focal lesion is accompanied by structural reorganization in the adult rat. Brain Struct Funct 218:437-453.

\section{Figures}

\section{Lesion/sham Handling Open field Fear conditioning}

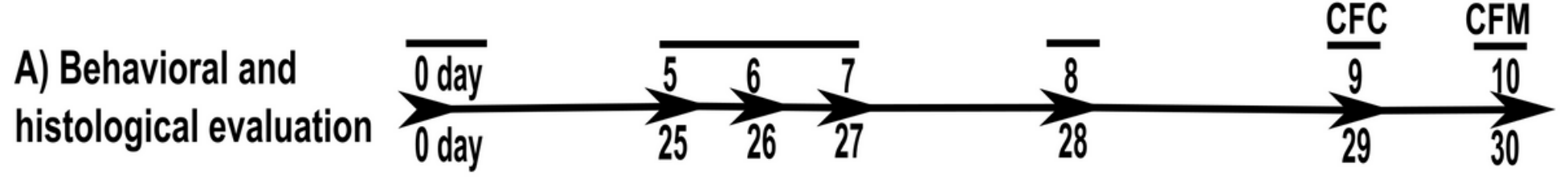

\section{B) Electrophysiological} recordings

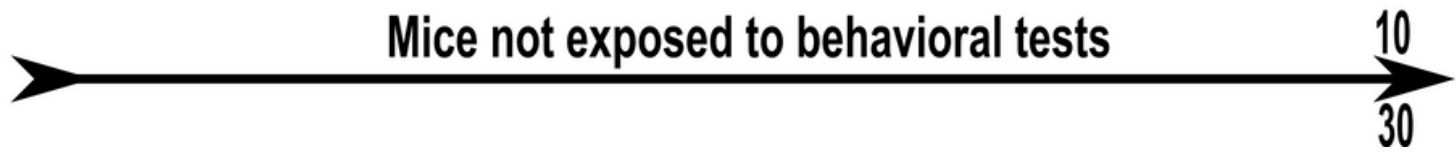

Figure 1

Experimental design. Kainic acid injections or sham surgeries were performed on day 0 . Mice were handled and habituated to the experimenter for three consecutive days before the onset of behavioral procedures. On days 8 or 28 after surgery, mice performed the open field task. The next day (day 9 or 29) mice underwent contextual fear conditioning (CFC), and $24 \mathrm{~h}$ later, contextual fear memory (CFM) was evaluated. After memory retrieval, subjects were sacrificed and brains were extracted for histological analysis. A different group of animals, not exposed to behavioral evaluation, was used for in vivo LTP recordings. 

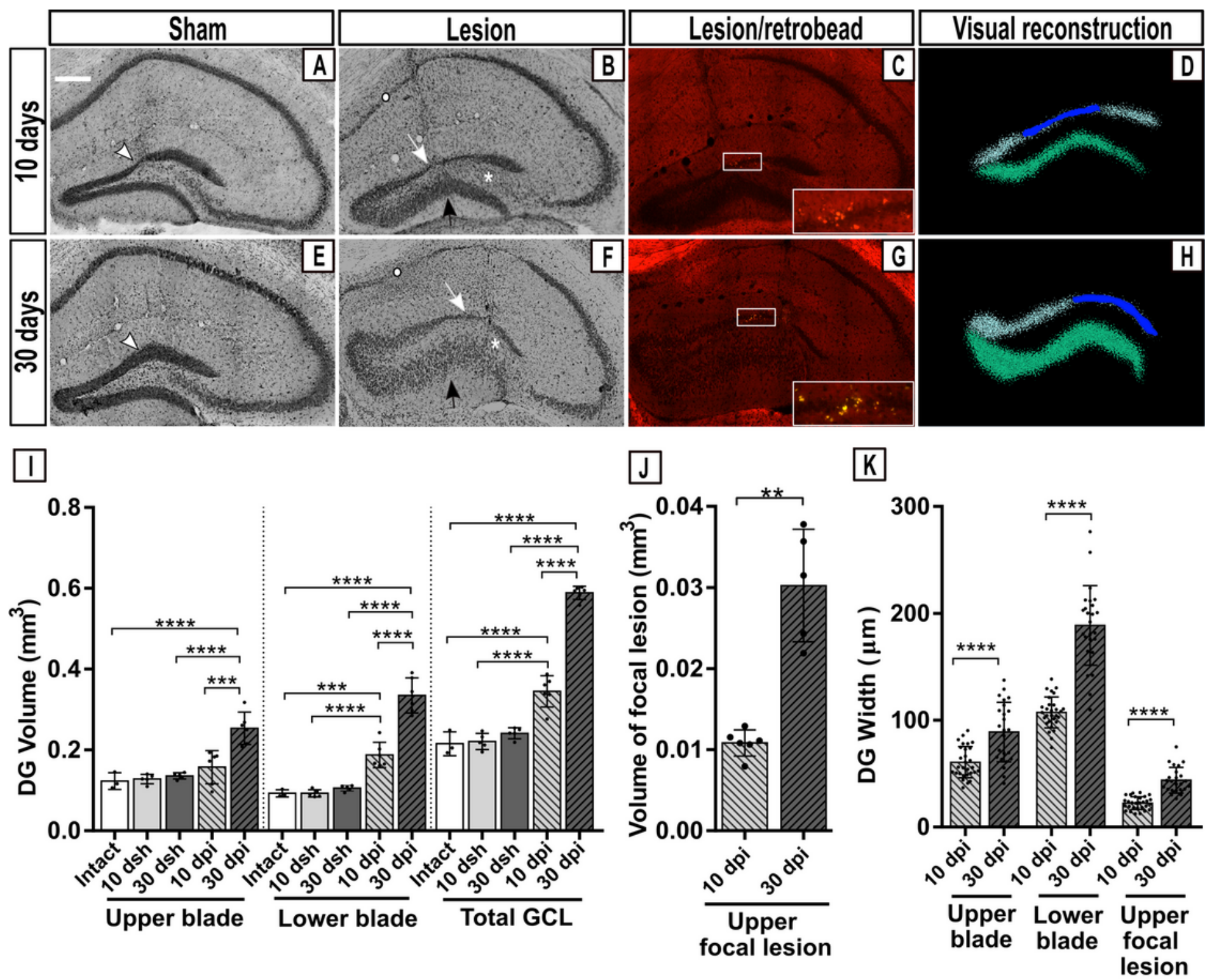

\begin{tabular}{|c|c|c|c|c|c|c|c|c|}
\hline$\square$ & $\begin{array}{c}\text { Upper blade } \\
\left(\mathrm{mm}^{3}\right)\end{array}$ & $\begin{array}{c}\text { Gundersen's } \\
\text { coefficient }\end{array}$ & $\begin{array}{c}\text { Lower blade } \\
\left(\mathrm{mm}^{3}\right)\end{array}$ & $\begin{array}{c}\text { Gundersen's } \\
\text { coefficient }\end{array}$ & $\begin{array}{c}\text { Total GCL } \\
\left(\mathrm{mm}^{3}\right)\end{array}$ & $\begin{array}{c}\text { Gundersen's } \\
\text { coefficient }\end{array}$ & $\begin{array}{c}\text { Upper lesion } \\
\left(\mathrm{mm}^{3}\right)\end{array}$ & $\begin{array}{c}\text { Gundersen's } \\
\text { coefficient }\end{array}$ \\
\hline Intact & 0.1229 & 0.0160 & 0.0927 & 0.0180 & 0.2156 & 0.0170 & --- & --- \\
\hline Sham 10d & 0.1281 & 0.0156 & 0.0928 & 0.0172 & 0.2209 & 0.0164 & --- & --- \\
\hline Sham 30d & 0.1357 & 0.0158 & 0.1054 & 0.0164 & 0.2411 & 0.0161 & --- & --- \\
\hline Lesion 10d & 0.1576 & 0.0165 & 0.1877 & 0.0173 & 0.3452 & 0.0169 & 0.0108 & 0.0423 \\
\hline Lesion 30d & 0.2540 & 0.0148 & 0.3092 & 0.0158 & 0.5632 & 0.0154 & 0.0302 & 0.0280 \\
\hline
\end{tabular}

Figure 2

Morphological changes in the DG over time after KA injection. Left images show representative Nisslstained coronal sections of the hippocampus from mice at (A) $10 \mathrm{dsh},(\mathbf{B}) 10 \mathrm{dpi},(\mathbf{E}) 30 \mathrm{dsh}$, and (F) 30 dpi. (A) At 10 dsh, sections display a mechanical lesion in the upper blade of DG as result of the cannula insertion (head arrow); scale bars: $300 \mu \mathrm{m}$. (E) At 30 dsh, the mechanical lesion is no longer evident (head arrow). (B) At $10 \mathrm{dpi}$, a focal lesion zone in the upper blade characterized by a thinning of the granule cell 
layer is observed (white arrow). Outside this lesion area, the upper blade shows GCL widening. (F) At 30 dpi, both DG blades display a more severe widening compared to $10 \mathrm{dpi}$. The black arrows indicate the prominent widening in lower blade. The white circle and the asterisk symbols indicate cell loss in CA1 and in the hilus, respectively. (C-G) The site where KA spread in the upper blade as revealed by the fluorescent tracer; insets show 40X magnifications. (D-H) A visual model of the DG based on Nissl-stained coronal sections: upper blade in gray, lesion site in blue, and lower blade in green. (I) The volume of the DG based on Cavalieri analysis (Intact $n=3 ; 10$ dsh $n=5 ; 30$ dsh $n=5 ; 10$ dpi $n=6 ; 30$ dpi $n=5$; one-way ANOVA followed by a Bonferroni's post hoc test, $\left.F_{(14,60)}=123.7, p<0.0001\right)$. (J) The volume from the lesion site in the upper blade from KA-injected mice (10 dpi $n=6 ; 30 \mathrm{dpi} n=5$; Unpaired t-test with Welch's correction, $F$ $(4,5)=18.31, p<0.0069)$. (K) GCL width from each DG section analyzed in KA-injected mice (10 dpi $n=29$ sections from 6 mice; 30 dpi $n=24$ sections from 5 mice; Unpaired t-test with Welch's correction; Upper blade, $F_{(23,28)}=3.625, p=0.0015$; Lower blade, $F_{(24,28)}=6.493, p<0.0001$; Upper blade lesion, $F_{(23,28)}=$ $3.962, p=0.0007)$. (L) Average volume and Gundersen's coefficient after a stereological Cavalieri analysis from the same mice used in histograms I-J; statistical significances are indicated in histograms I-J. A Gundersen's coefficient less than 0.1 means statistically valid volume of data. The columns represent the mean SD from each group. Dots superimposed in the graph represent individual values. Asterisks indicate statistically significant differences $\left(p^{*}<0.05 ; p^{\star \star}<0.01 ; p^{\star \star \star}<0.001 ; p^{\star \star \star \star}<0.0001\right)$. 

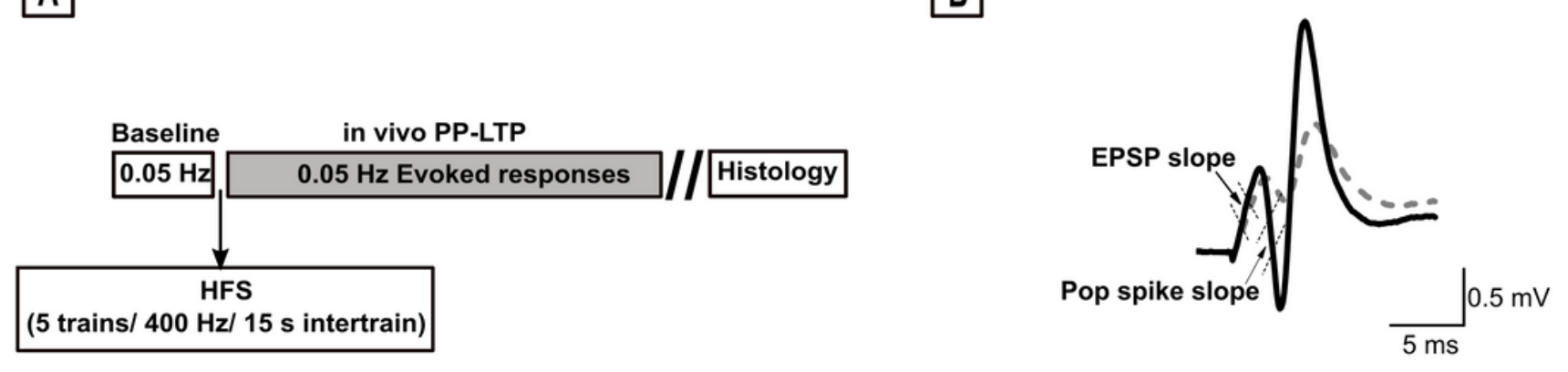

C

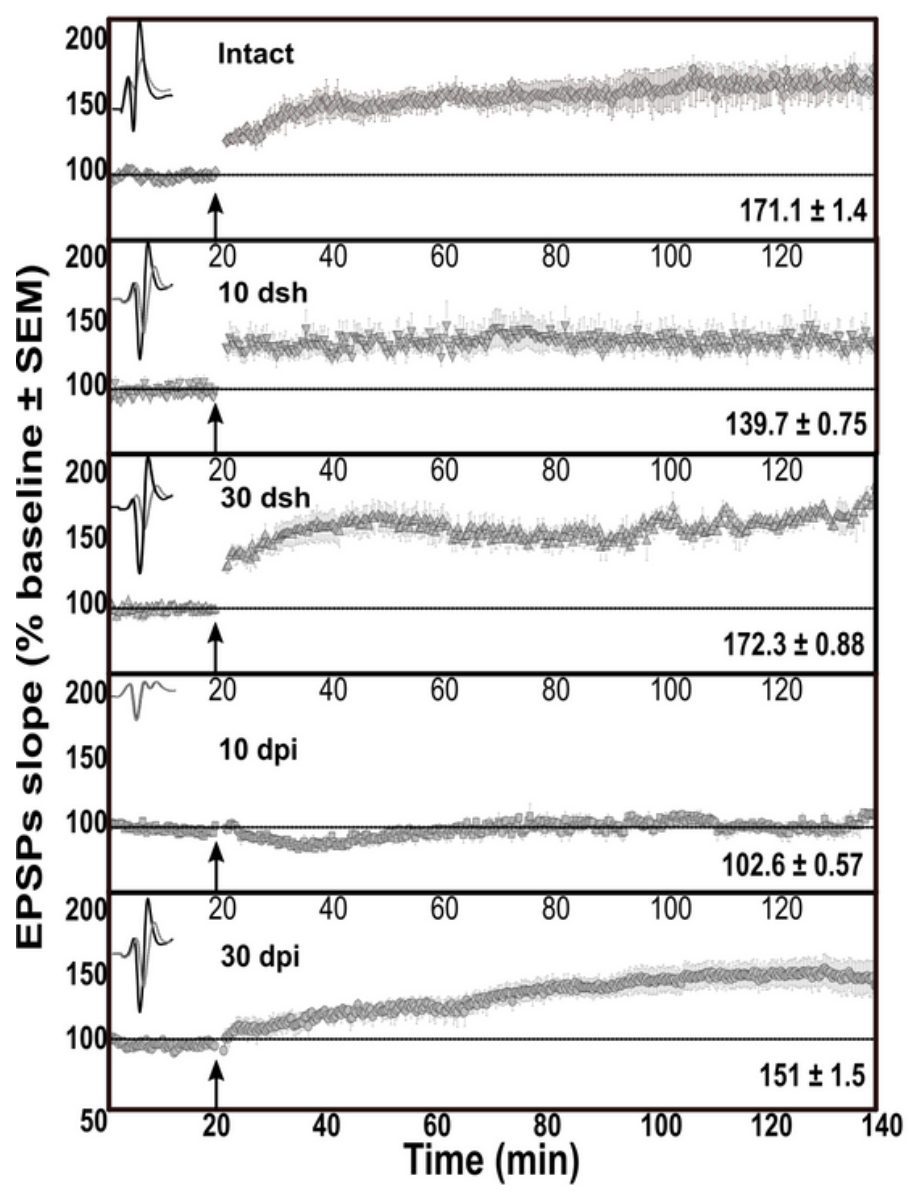

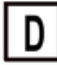

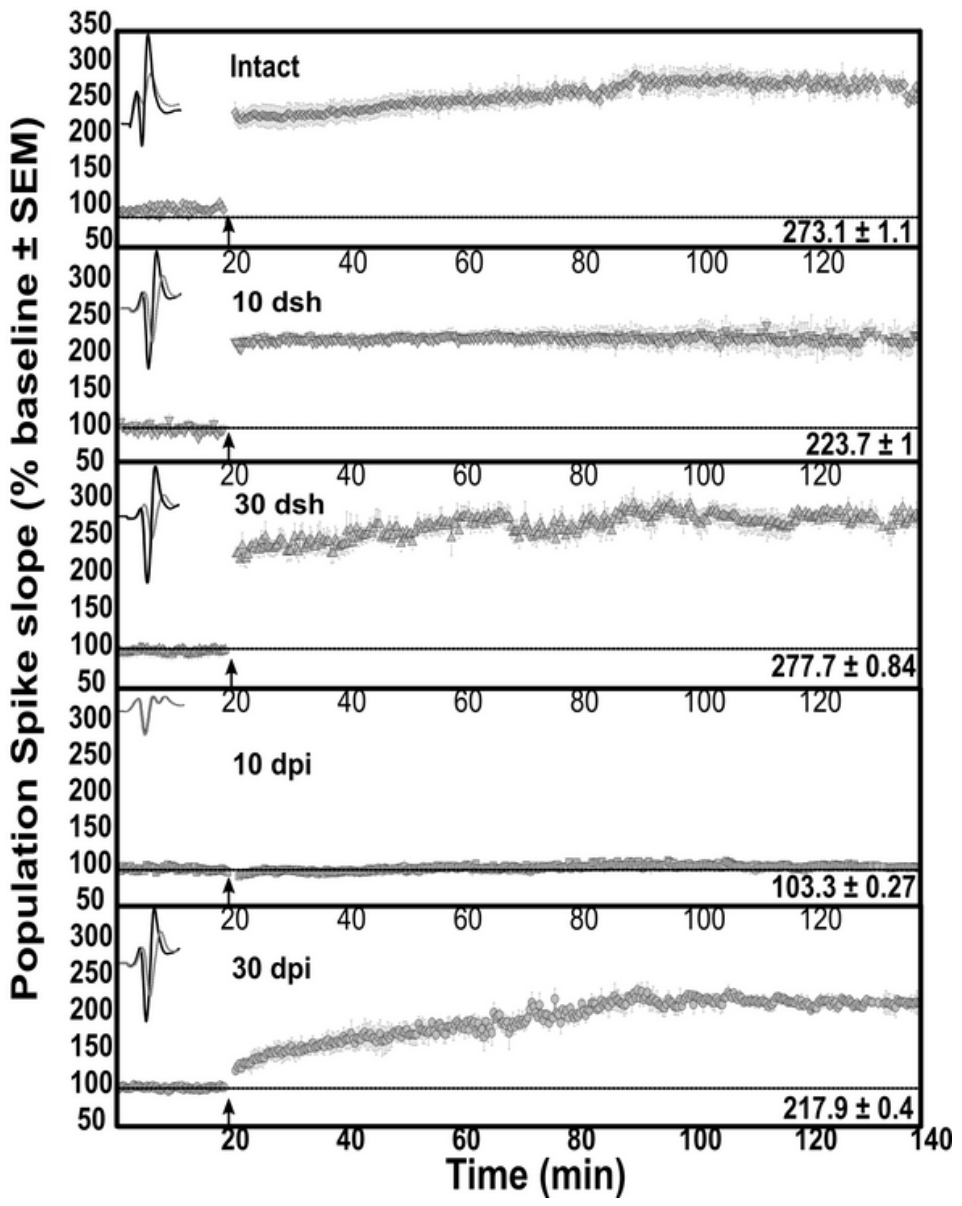

Figure 3

KA-induced alterations in the DG modifies in vivo perforant pathway LTP along time. (A) Schematic representation of experimental procedures. Conditioning stimulus trains began after $20 \mathrm{~min}$ of baseline recording and were maintained for $120 \mathrm{~min}$. (B) Representative traces of the excitatory post-synaptic potential (EPSP) obtained during baseline (dotted line) and $140 \mathrm{~min}$ after stimulation (full line). (C) Plot of the EPSP slope for the last 20 min after high-frequency stimulation. Intact and 30 dsh groups displayed similar slope percentages of EPSP (intact, 171.1\%; 30 dsh 172.3\%).The EPSP slope percentage in the $10 \mathrm{dsh}$ group, was $139.7 \%$, that is, less than intact and $30 \mathrm{dsh}$ group. In the $10 \mathrm{dpi}$ group, LTP induction was impaired, as opposed to the $30 \mathrm{dpi}$ group where LTP induction was recovered. Notably, LTP from mice at $30 \mathrm{dpi}$ took longer time to reach its maximal response compared to all other groups. All 
these findings are more evident in the population spike slope percentage (D). Inner numbers show the mean \pm SEM of slope (percent of baseline) from the last 20 min of recording. Application of conditioning stimulus trains is indicated (arrow). Repeated-measures ANOVA followed post hoc Fisher's test; intact $n=4 ; 10$ dsh $n=4 ; 30$ dsh $n=4 ; 10$ dpi $n=4 ; 30$ dpi $n=4$.
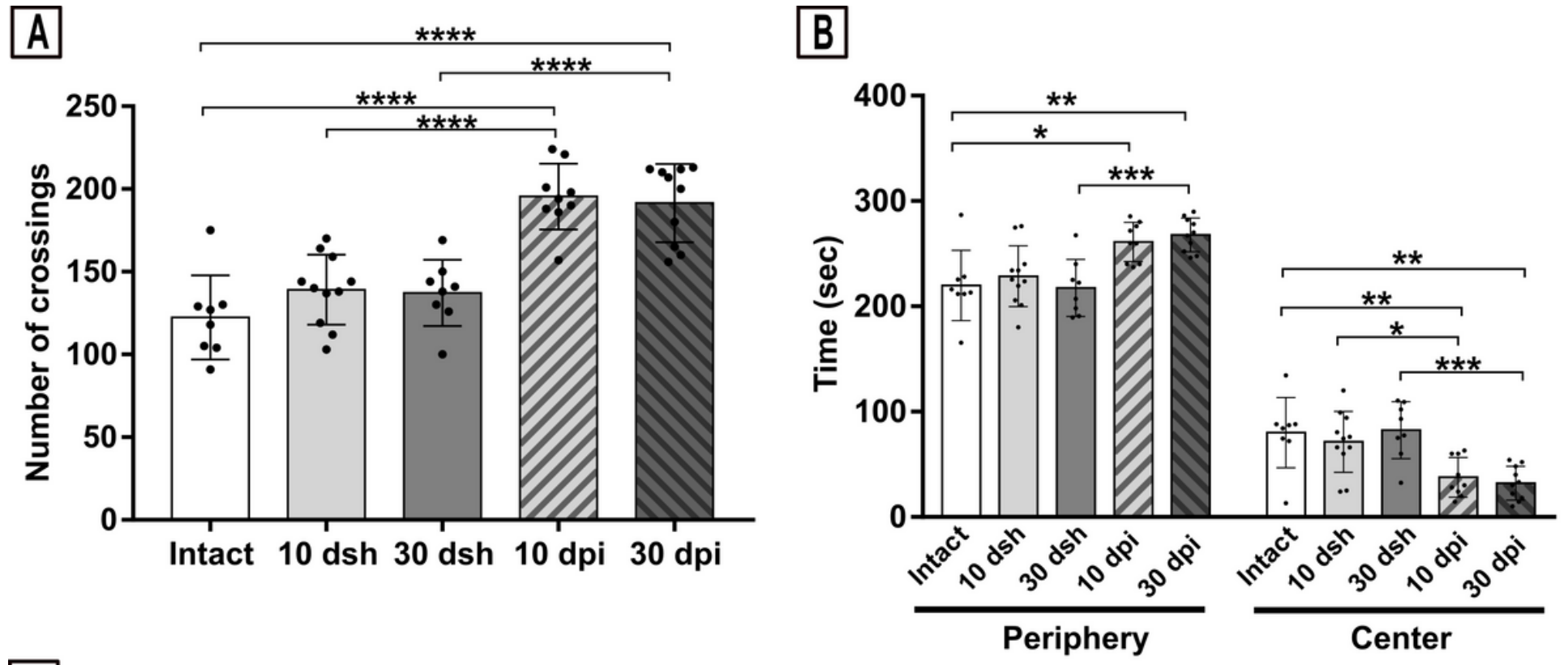

C

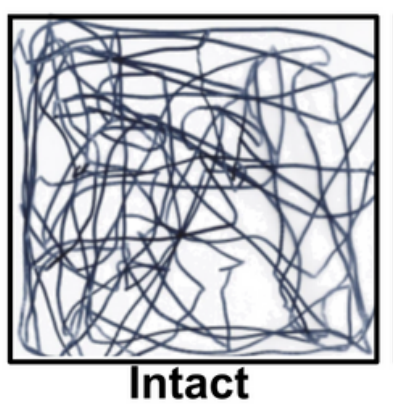

Intact

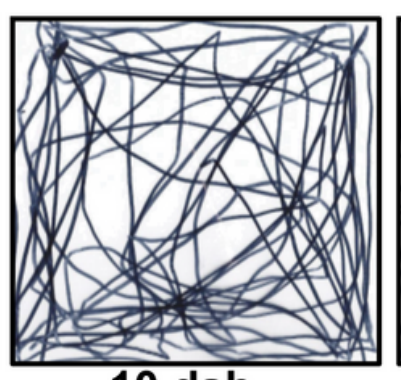

$10 \mathrm{dsh}$

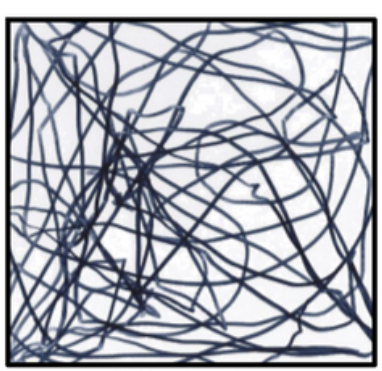

$30 \mathrm{dsh}$

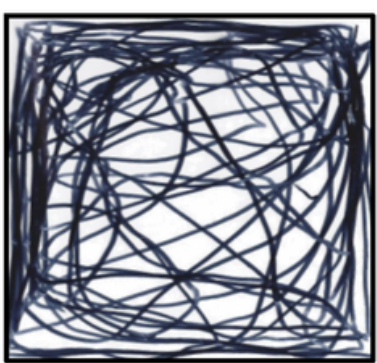

$10 \mathrm{dpi}$

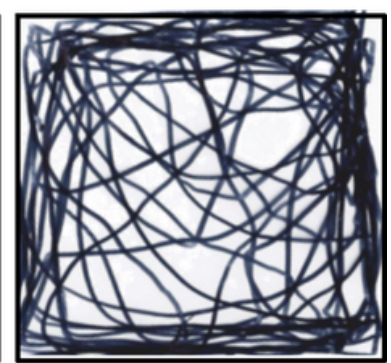

$30 \mathrm{dpi}$

Figure 4

Mice at $10 \mathrm{dpi}$ and $\mathbf{3 0} \mathrm{dpi}$ show increased motility and anxiety like behavior. (A) Motor behavior was evaluated through the number of crossings. KA-injected mice at 10 and $30 \mathrm{dpi}$ show a higher number of crossings than intact and sham mice (one-way ANOVA followed by a Bonferroni's post hoc test, $F_{(4,41)}=$ $21.2, p<0.0001)$. (B) Anxiety-like behavior was evaluated through the time spent in the periphery and center zones of the field. KA-injected mice at 10 and 30 dpi spent more time on periphery and less time on center zones than intact and sham mice (two-way ANOVA followed by a Bonferroni's post hoc test, interaction $\left.F_{(4,82)}=16.18, p<0.0001\right)$. (C) Representative path length tracks from a mouse from each group are shown. The columns represent the mean with SD from each group. Dots superimposed in the graph represent individual values. Asterisks indicate statistically significant differences $\left(p^{\star} \leq 0.05 ; p^{\star *}<\right.$ $\left.0.01 ; p^{\star \star *}<0.001 ; p^{\star \star \star *}<0.0001\right)$. Intact $n=8,10$ dsh $n=11,30$ dsh $n=8,10$ dpi $n=9,30$ dpi $n=10$. 

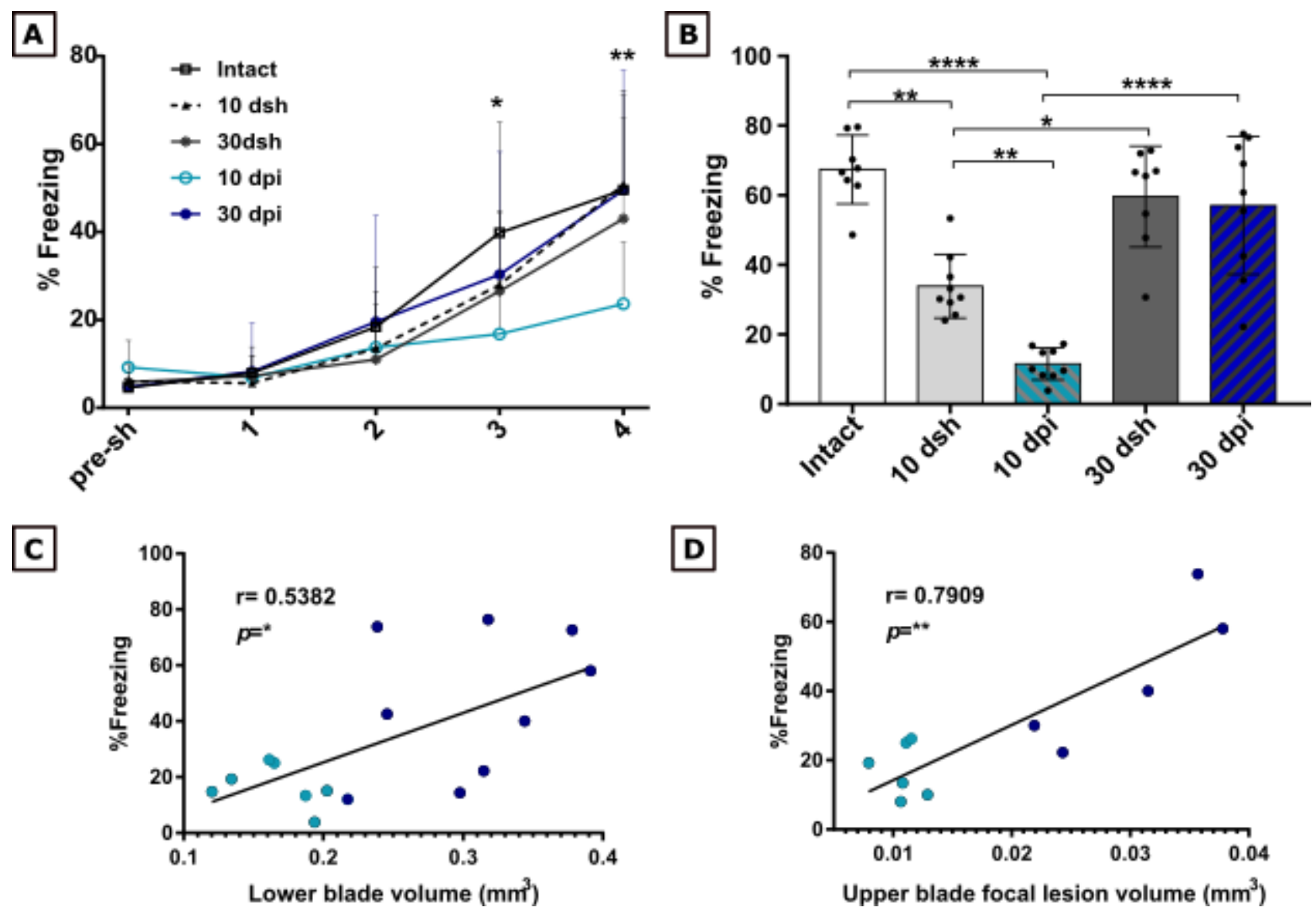

Figure 5

Contextual fear memory was impaired at 10 dpi but restored at 30dpi. (A) Learning curve for contextual fear conditioning. All groups, except mice at $10 \mathrm{dpi}$, showed progressive learning reflected by the increase in the percentage of freezing time across trials. Mice at 10 dpi show lower freezing percentage time than intact mice on trials 3 and 4. Points represent the mean with SD for each group across trials (Asterisks denote differences from intact vs 10dpi; two-way ANOVA followed by a Bonferroni's post hoc test, ( $F$ $(4,192)=43.07, p<0.0001)$. (B) Contextual fear memory retrieval. Mice at $10 \mathrm{~d}$ sh show lower freezing than intact mice, but more freezing than mice at $10 \mathrm{dpi}$. At $30 \mathrm{dpi} \mathrm{mice} \mathrm{show} \mathrm{similar} \mathrm{freezing} \mathrm{as} \mathrm{intact} \mathrm{and} \mathrm{as}$ 30 dsh mice. The columns represent mean and SD for each group. Dots superimposed on the graph represent individual values (one-way ANOVA followed by a Bonferroni's post hoc test, $F_{(4,38)}=28.29$, $p<$ 0.0001). (C-D) Graph showing the positive correlation between the volume of lower blade (C, Spearman correlation coefficient, $r=0.5382 ; 95 \%$ confidence interval ( 0.04198 to 0.8215$) ; n=16$ ) and upper blade lesion ( $D$, Spearman correlation coefficient, $r=0.7909 ; 95 \%$ confidence interval $(0.3456$ to 0.9455$) ; n=11$ ) with the percentage of freezing in lesioned mice. For $\mathbf{A}-\mathbf{B}$, Intact $n=8,10$ dsh $n=9,30$ dsh $n=9,10$ dpi $n=9$, $30 \mathrm{dpi} n=9$. Asterisks indicate statistically significant differences $p^{*} \leq 0.05 ; p^{\star \star} \leq 0.01 ; p^{\star \star \star} \leq 0.001$. 